\title{
Insuficiencia aórtica severa secundaria a artritis reumatoide con choque cardiogénico y muerte
}

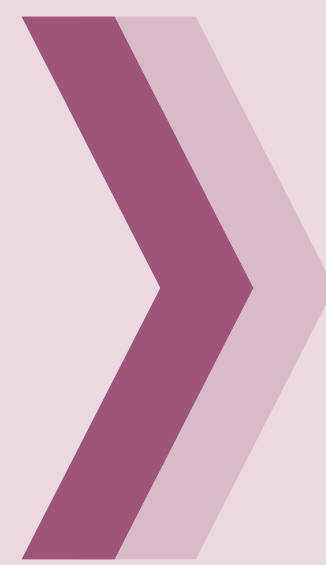

\section{Severe aortic regurgitation caused by rheumatoid arthritis with cardiogenic shock and death: Report of a case}

\section{Alex Gallegos-Cazorla}

1. Médico cardiólogo. Hospital Nacional Daniel Alcides Carrión, Callao. Profesor de la Facultad de Medicina Humana de la Universidad de San Martín de Porres. Maestro en investigación y docencia universitaria.

\section{RESUMEN}

La artritis reumatoide (AR) afecta frecuentemente al corazón produciendo lesiones valvulares hemodinámicamente no significativas. La insuficiencia aórtica severa secundaria es rara; sin embargo, puede progresar rápidamente $y$, si no es advertida a tiempo, pone en peligro la vida del paciente. Presentamos el caso de una mujer de 45 años con antecedente de artritis reumatoide, diagnosticada desde los 25 años, quien fue admitida al hospital Daniel Alcides Carrión, del Callao, por choque cardiogénico y AR no activa. Refirió dos semanas de tos seca, disnea al reposo e intolerancia al decúbito, que se incrementó progresivamente. Ingresó con PA de 60/30 mm Hg. El examen cardiovascular evidenció soplo diastólico regurgitativo aórtico IIVI, soplo sistólico eyectivo aórtico II/VI, con pulso filiforme y de Corrigan. El electrocardiograma mostró hipertrofia ventricular izquierda. La ecocardiografía reportó cardiopatía dilatada con insuficiencia aórtica severa, disfunción sistólica moderada e hipertensión pulmonar moderada. El choque fue refractario al tratamiento y la paciente falleció en asistolia a los 10 días de su ingreso.

Palabras clave: Insuficiencia de la válvula aórtica; Artritis reumatoide; Válvula aórtica; Choque cardiogénico

\begin{abstract}
Rheumatoid arthritis (RA) frequently affects the heart with no hemodynamically significant valvular lesions. Severe secondary aortic regurgitation is rare, however it can progress rapidly and if not noticed in time endangers the patient's life. We present the case of a 45 year old woman with a history of $R A$ diagnosed since age 25, admitted to hospital Daniel Alcides Carrión in Callao due to cardiogenic shock and RA not active. She reported two weeks of dry cough, dyspnea, and decubitus intolerance which progressively increased. Aortic diastolic murmur II / VI and aortic systolic ejection murmur II / VI were auscultated with thready Corrigan's pulse. At admission BP was $60 / 30 \mathrm{mmHg}$. Electrocardiogram showed left ventricular hypertrophy. Echocardiography reported dilated left ventricle with severe aortic regurgitation, moderate systolic dysfunction and moderate pulmonary hypertension. Shock was refractory to treatment and patient died 10 days after admission.
\end{abstract}

Key words: Aortic valve insufficiency; Arthritis rheumatoid; Aortic valve; Shock cardiogenic 


\section{INTRODUCCIÓN}

La artritis reumatoide (AR) a menudo cursa con anormalidades cardiovasculares estructurales o funcionales que llegan a comprometer las válvulas cardiacas. La valvulopatía asociada con AR cursa, la mayoría de las veces, inactiva; sin embargo, algunos pacientes desarrollan insuficiencia rápidamente progresiva con deterioro hemodinámico y desenlace fatal. Debido a que son infrecuentes las disfunciones valvulares significativas, no hay recomendaciones específicas para su detección o tratamiento. El reporte del caso muestra las complicaciones cardiovasculares de la AR. La detección temprana de síntomas de falla cardiaca, el examen físico cardiovascular, los exámenes de ayuda diagnóstica (ecocardiografía) y la sumatoria del cardiólogo al tratamiento multidisciplinario de la AR, pueden evitar la progresión de la enfermedad valvular cardiaca hasta la muerte.

\section{REPORTE DEL CASO}

Paciente mujer de 45 años, con diagnóstico de AR desde los 25 años de edad, es admitida al hospital Daniel Alcides Carrión, del Callao, con choque cardiogénico. Refirió dos semanas de tos seca, disnea al reposo e intolerancia al decúbito, que se incrementó progresivamente. Al ingreso se registró PA de 60/30 mmHg; FC: 100 lpm; FR: 26 rpm. Tórax: roncantes difusos. Aparato cardiovascular: soplo sistólico regurgitativo aórtico II/VI, soplo sistólico eyectivo aórtico II/VI y pulso filiforme y saltón. Fue evaluada por el reumatólogo, quien diagnosticó AR no activa y reportó cambios degenerativos articulares característicos de la enfermedad.
El electrocardiograma (Figura 1) mostró hipertrofia ventricular izquierda, con taquicardia sinusal y trastorno de la repolarización, sin signos de evento coronario agudo. La radiografía de tórax no mostró foco neumónico, ni signos de fibrosis pulmonar secundarios a AR. La ecocardiografía reportó insuficiencia aórtica severa (Figuras 2 y 4), dilatación ventricular izquierda severa, con $64,4 \mathrm{~mm}$ de diámetro telediastólico (Figura 5); hipertensión pulmonar moderada, $63 \mathrm{mmHg}$, (Figura 6) y disfunción sistólica moderada, con fracción de eyección 43\% (Figura 7), relacionados con la cronicidad de la insuficiencia aórtica.

Tratada en $\mathrm{UCl}$ con vasoactivos, inotrópicos y soporte ventilatorio, continuó con choque refractario; se agregaron neumonía e insuficiencia renal aguda. Falleció a los 10 días de su ingreso; no se realizó la necropsia.

\section{DISCUSIÓN}

La $A R$ es una enfermedad sistémica con trastornos inflamatorios autoinmunitarios, que con frecuencia producen alteraciones en el corazón. La cardiopatía puede producirse en pacientes con un diagnóstico ya establecido de un trastorno reumático. La afección cardiaca en la AR puede variar entre asintomática o leve y grave o peligrosa para la vida. Los pacientes pueden no presentar síntomas cardiacos clínicos manifiestos, lo que hace que el diagnóstico de la cardiopatía resulte más difícil. Los síntomas son una indicación importante para el reemplazo valvular aórtico en los pacientes con una insuficiencia aórtica severa. Los pacientes con insuficiencia aórtica severa crónica que desarrollan síntomas tienen un

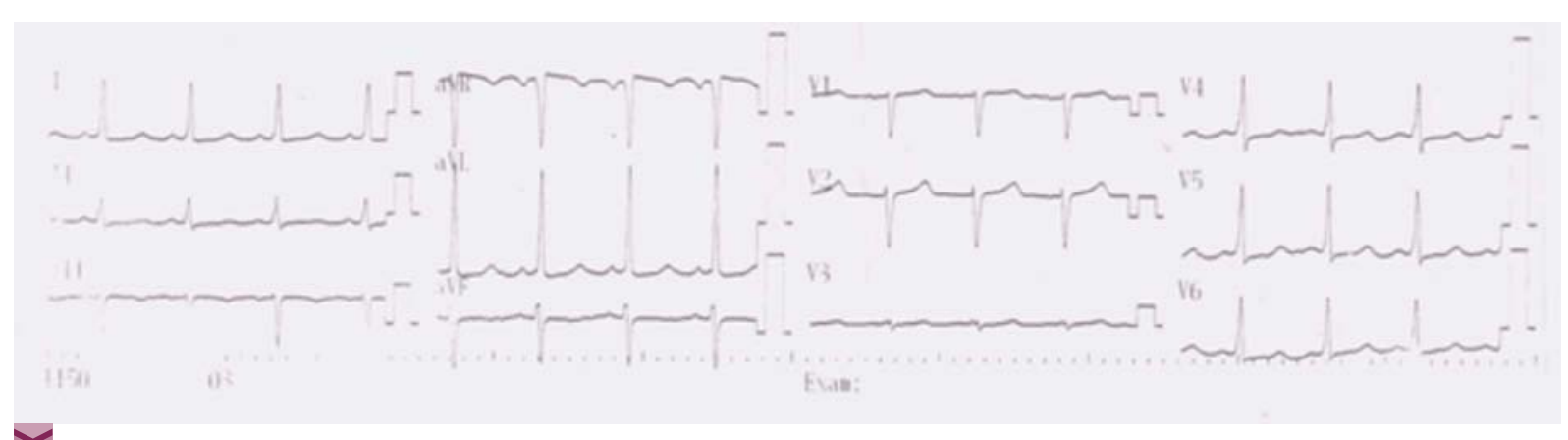

Figura 1. Electrocardiograma con HVI 


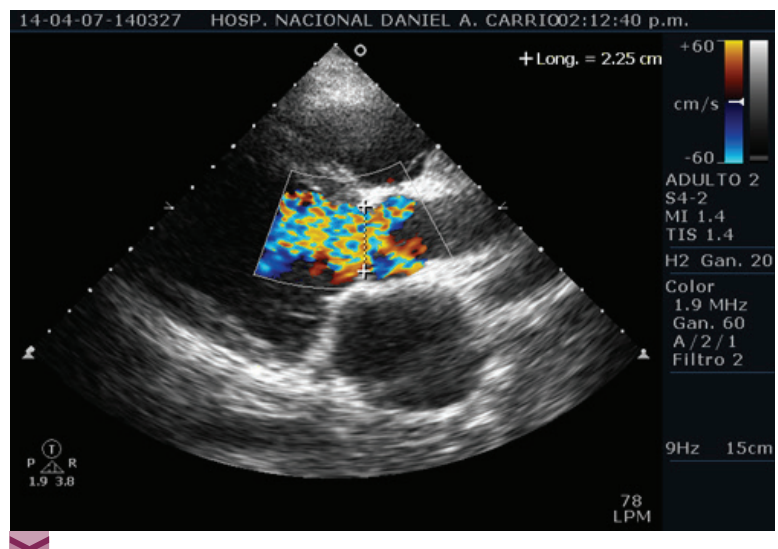

Figura 2. Ecocardiografía para esternal eje largo y doppler color de válvula aórtica con insuficiencia severa

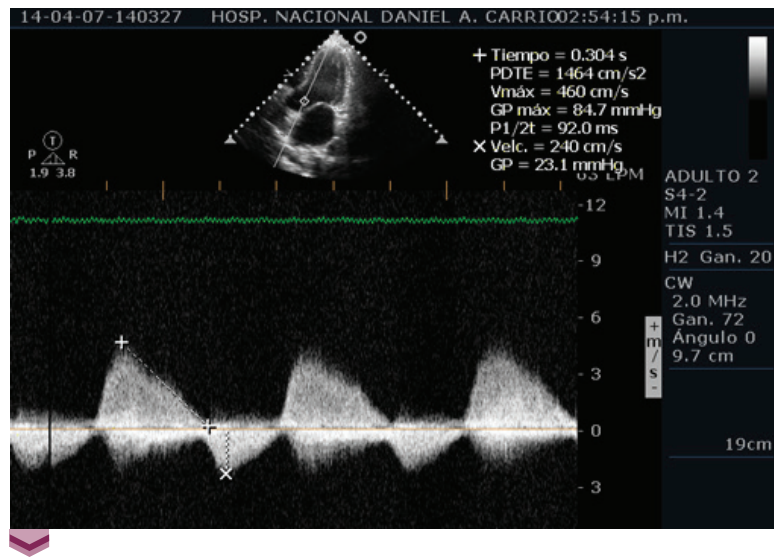

Figura 4. Ecocardiografía doppler contínuo de valvula aótica con insuficiencia severa

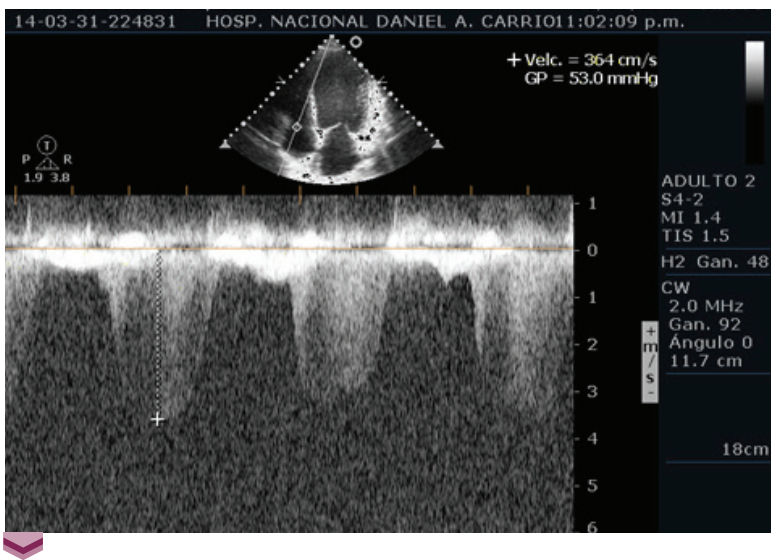

Figura 6. Ecocardiografía doppler contínuo de valvula tricúspide con hipertensión pulmonar moderada

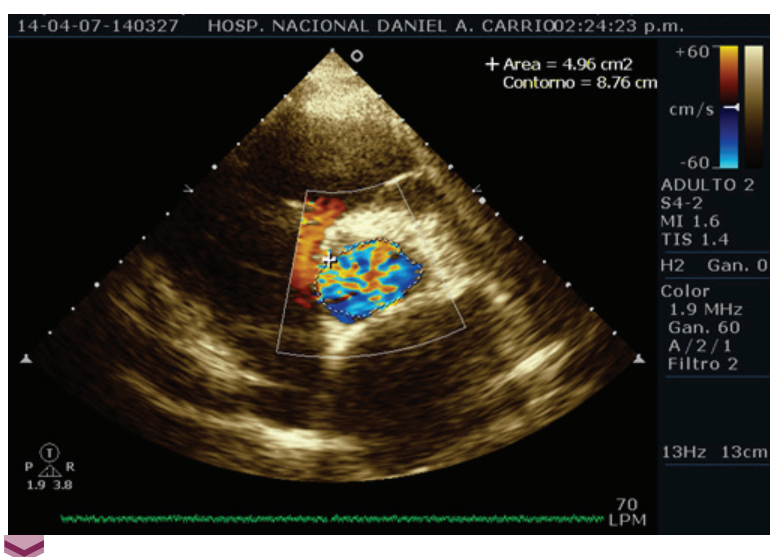

Figura 3. Ecocardiografía para esternal eje corto y doppler color de válvula aórtica con insuficiencia severa

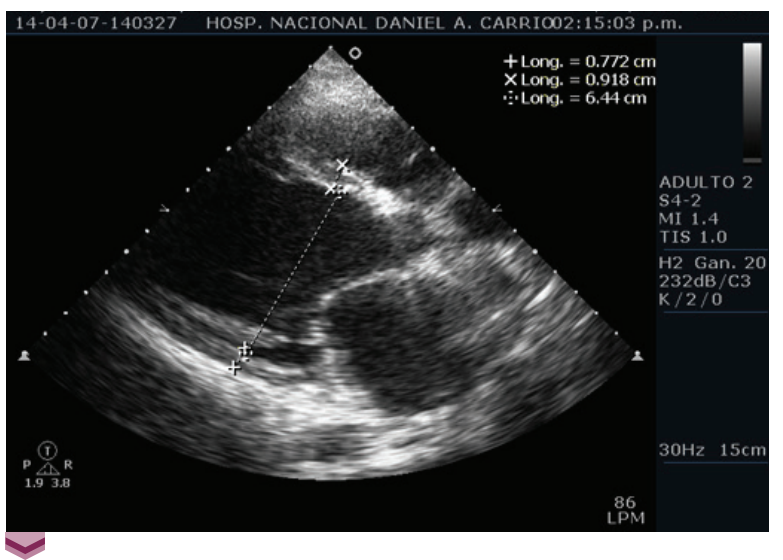

Figura 5. Ecocardiografía con dilatación del ventrículo izquierdo

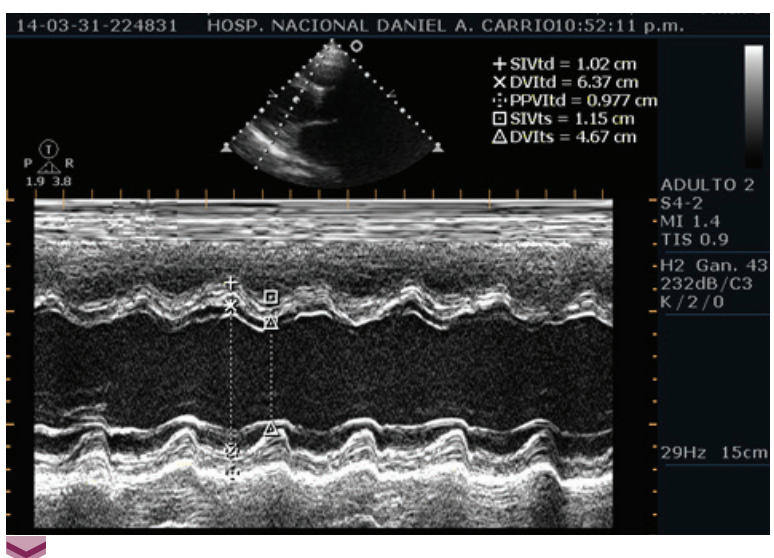

Figura 7. Ecocardiografía con disfunción sistólicaventricular izquierda moderada 
alto riesgo de morir si no se realiza el reemplazo valvular aórtico; la mortalidad en estos pacientes sin tratamiento quirúrgico puede ser tan alta como $10-20 \%$ anualmente Así, tenemos que en una serie de 246 pacientes con insuficiencia aórtica severa seguidos sin cirugía, aquellos que estuvieron en clase III-IV NYHA tuvieron una tasa de mortalidad de $24,6 \%$ por año. La importancia de los síntomas preoperatorios al reemplazo valvular se observó en pacientes con disfunción sistólica ventricular izquierda. ${ }^{1}$

La AR tiene predominancia femenina y una prevalencia del $1 \%$, que se incrementa con la edad. ${ }^{2}$ En el año 2013 se realizó una revisión sistemática con un metaanálisis acerca del compromiso valvular y pericárdico, en la que se encontró que la AR estuvo significativamente asociada con insuficiencia valvular aórtica; se concluyó en la importancia de la realización de una evaluación ecocardiográfica. La AR causa destrucción valvular ocasionando tanto estenosis como insuficiencia valvular. ${ }^{3}$ El caso presentado es el de una paciente con AR de 20 años de evolución. Si bien es cierto, la AR no fue diagnosticada como activa clínicamente, su larga evolución y el compromiso cardiaco evidenciado por la ecocardiografía sustentan altamente la relación con el tiempo; más aún, al no haber otra causa probable (e.g., aneurisma de aorta).

La paciente estuvo postrada y en situación de reposo casi permanente por las lesiones articulares secundarias a $A R$, lo que, probablemente, hizo que los síntomas no se manifiesten hasta etapas avanzadas de la enfermedad. Estudios ecocardiográficos y de autopsia revelaron anormalidades valvulares hasta en un $70 \%$ de los pacientes con $\mathrm{AR}^{4}$ pero, usualmente, no hay repercusión clínica. Algunos pocos pacientes pueden desarrollar insuficiencia valvular, donde la válvula aórtica es la más afectada. 5 Las lesiones valvulares más características de la AR son los granulomas reumatoideos, los cuales se encuentran en un 3 a 5\% en las autopsias; 6 pero, esto generalmente no tiene trascendencia clínica ni hemodinámica.

La alteración en la válvula aórtica produce insuficiencia que, en general, no es significativa y que fue encontrada en 1 a $3 \%$ de los antiguos estudios, si bien las formas menores y asintomáticas son más frecuentes en los estudios ecográficos recientes. Las insuficiencias aórticas de origen reumatoideo, operadas en general, se observan en forma aislada y su gravedad evolutiva, puede comprometer rápidamente la vida. ${ }^{7}$

La paciente presentó en su ecocardiografía dilatación ventricular izquierda con sobrecarga severa de volumen en el ventrículo izquierdo, a lo que se agregó hipertensión arterial pulmonar moderada; todo esto demuestra una evolución crónica de la enfermedad. En los pacientes asintomáticos con insuficiencia aórtica severa crónica y función ventricular izquierda normal, la posibilidad de eventos adversos es baja. Sin embargo, cuando el diámetro telediastólico del ventrículo izquierdo es mayor de $50 \mathrm{~mm}$, la posibilidad de muerte, síntomas o disfunción ventricular izquierda llega a ser de 19\% anualmente. ${ }^{8-10}$ No se llegó a visualizar granulomas valvulares que, por lo demás, son infrecuentes. La ecocardiografía transesofágica pudo haber precisado el daño valvular con mayor definición; lamentablemente, su estado de choque no hizo posible el procedimiento.

El pronóstico de la insuficiencia aórtica de causa reumatoidea parece ser globalmente más grave que el de las insuficiencias de la válvula aórtica originadas en otras causas, lo que se podría demostrar por el hecho de que los reemplazos valvulares son, con frecuencia, necesarios, debido al rápido deterioro del estado hemodinámico. Su inestabilidad hemodinámica y el difícil acceso a la cirugía cardiaca no hizo viable este tratamiento Un estudio reportó un mayor número de anormalidades ecocardiográficas en pacientes con artritis nodular más severa (40\%) que con AR no nodular $(17,2 \%)$ o en los controles (14,3\%).11

La insuficiencia aórtica, en general, no se diagnostica y se observa con más frecuencia en las mujeres de más de 40 años que presentan formas graves de poliartritis reumatoidea, con serología reumatoidea positiva, evolución en los últimos diez años o menos y con manifestaciones extraarticulares habituales. Cuando se detecta clínicamente, ya es grave o mal tolerada, por lo que se requiere el rápido reemplazo valvular. La paciente se encontraba en el grupo etario más vulnerable a las formas graves de $A R$; sin 
embargo, la evaluación por el reumatólogo, que si bien encontró cambios degenerativos articulares característicos de $A R$, no concluyó en señalar que se trataba de una enfermedad reumática activa, por lo que la severidad de la insuficiencia probablemente estuvo dada por la propia historia natural de la enfermedad.

Existen varios casos reportados de insuficiencia aórtica causada por artritis reumatoide, ${ }^{12-14}$ no obstante, en casi todos los casos, la insuficiencia progresaba crónicamente sin llegar al choque cardiogénico, como en el presente caso. Aziz et al. ${ }^{15}$ reportaron un caso que mostró una progresión de la enfermedad en un mes, mientras que Mannaerts et al. ${ }^{16}$ reportaron un caso en el que la enfermedad progresó en dos años. Así mismo, Masami et al. reportaron recientemente un caso raro de progresión de la enfermedad en dos semanas. ${ }^{17}$ La paciente presentó un rápido deterioro hemodinámico, que llegó al choque cardiogénico.

La elevada mortalidad también está en relación con la disfunción sistólica que presentaba la paciente. En una serie de pacientes posoperados, de 450 pacientes sometidos a reemplazo valvular aórtico, aquellos con disfunción sistólica severa tuvieron una alta mortalidad a corto y largo plazo. Esto se confirmó en otro estudio de 724 pacientes, en los que la sobrevivencia se redujo significativamente con disfunción sistólica severa, comparados con aquellos de función normal o menos severa. ${ }^{18-20}$ Este el primer caso, hasta donde tenemos conocimiento, en el que se reporta el rápido y progresivo deterioro de una insuficiencia valvular aórtica, probablemente crónica, no asociada con la exacerbación de su enfermedad reumatoidea.

\section{CONCLUSIONES}

Se presenta un caso inusual de insuficiencia aórtica, probablemente inducida por artritis reumatoide, que evolucionó con choque cardiogénico y luego deceso. Se debe reconocer a la AR como un importante factor de riesgo de deterioro valvular cardiaco, que puede llevar a la muerte. El tratamiento de los pacientes reumatológicos debe ser multidisciplinario; se recomienda involucrar al especialista en cardiología.

\section{REFERENCIAS BIBLIOGRÁFICAS}

I. Dujardin KS, Enriquez-Sarano M, Schaff HV, Bailey KR, Seward JB, Tajik AJ. Mortality and morbidity of aortic regurgitation in clinical practice. A long-term follow-up study. Circulation. 1999;99(|4): 1851-7.

2. Lawrence RC, Helmick CG, Arnett FC, Deyo RA, Felson DT, Giannini EH. Estimates of the prevalence of arthritis and selected musculoskeletal disorders in the United States. Arthritis Rheum. 1998;4I(5):778-99.

3. Corrao S, Messina S, Pistone G, Calvo L, Scaglione R, Licata G. Heart involvement in rheumatoid arthritis: systematic review and meta-analysis. Int J Cardiol. 20I3;167(5):203I-8.

4. Kitas G, Banks MJ, Bacon PA. Cardiac involvement in rheumatoid disease. Clin Med. 200I;I(I):I8-2I.

5. González T: Artritis reumatoidea, manifestaciones extraarticulares. En: Andreu JL, Barceló P, Figueroa M, HerreroBeaumont G, Martín Mola E, Olivé A, et al, editores. Manual de enfermedades reumáticas de la Sociedad Española de Reumatología. Madrid: Mosby/Doyma; 1996. p. 316-23.

6. Carpenter DF, Golden A, Roberts WC. Quadrivalvular rheumatoid heart disease associated with left bundle branch block.Am J Med. 1967;43(6):922-9.

7. Baldé MD, Ecke JE, Ortega L, Caulet-Maugendre LS, Chabanne C, Almange C, Daubert JC, Leguerrier A, Laurent M. Valve replacement for aortic regurgitation associated with rheumatoid arthritis: a series of 5 cases. Arch Mal Coeur Vaiss. 2006;99(6):569-74.

8. Bonow RO, Lakatos E, Maron BJ, Epstein SE. Serial long-term assessment of the natural history of asymptomatic patients with chronic aortic regurgitation andnormal left ventricular systolic function. Circulation. 199|;84(4): I625-35.

9. Klodas E, Enriquez-Sarano M, Tajik AJ, Mullany CJ, Bailey KR, Seward JB. Optimizing timing of surgical correction in patients with severe aortic regurgitation: role of symptoms. J Am Coll Cardiol. 1997;30(3):746-52.

10. Dujardin KS, Enriquez-Sarano M, Schaff HV, Bailey KR, Seward JB, Tajik AJ. Mortality and morbidity of aortic regurgitation in clinical practice. A long-term follow-up study. Circulation. 1999;99(|4):185|-7.

I I. Wisłowska M, Sypuła S, Kowalik I. Echocardiographic findings and 24-h electrocardiographic Holter monitoring in patients with nodular and non-nodular rheumatoid arthritis. Rheumatol Int. 1999;18(5-6):163-9.

12. Roldan CA, DeLong C, Qualls CR, Crawford MH. Characterization of valvular heart disease in rheumatoid arthritis by transesophageal echocardiography and clinical correlates. Am J Cardiol. 2007; 100(3):496-502.

13. Uusimaa P, Krogerus ML, Airaksinen J, Linnaluoto M, Tervonen $O$, Hakala M. Aortic valve insufficiency in patients with chronic rheumatic diseases. Clin Rheumatol. 2006;25(3):309-I3.

14. Minematsu N,Yoshikai M, Kamohara K, Tomimitsu S.Aortic valve regurgitation associated with rheumatoid arthritis; report of a case. Kyobu Geka. 2004;57(5):391-4.

15. Aziz S, Sohail M, Murphy G. Acute aortic regurgitation due to necrotizing granulomatous inflammation of the aortic valve in a patient with rheumatoid arthritis. Circulation. 20I2; 126(8):el06-7.

16. Mannaerts HF,May JF,Vierboom MA, Hamer JP. Rapidly progressive aortic insufficiency in a female patient with rheumatoid arthritis. Ned Tijdschr Geneeskd. 1994; 138(I2):618-2I.

17. Shingaki M, KobayashiY,Suzuki H.A case of acute aortic insufficiency due to severe rheumatoid arthritis, showing progression in two weeks. Cardiovasc Diagn Ther. 2014;4(3):267-9. 


\section{Anta Mérilica Pernana}

18. Forman R, Firth BG, Barnard MS. Prognostic significance of preoperative left ventricular ejection fraction and valve lesion in patients with aortic valve replacement. Am J Cardiol. 1980;45(6): I I20-5.

19. Bonow RO, Picone AL, Mclntosh CL, Jones M, Rosing DR, Maron $B$ J, et al.Survival and functional results after valve replacement for aortic regurgitation from 1976 to 1983: impact of preoperative left ventricular function. Circulation. 1985;72(6): I244-56.

20. Michel PL, lung B, Abou Jaoude S, Cormier B, Porte JM,Vahanian $A$, et al. The effect of left ventricular systolic function on long term survival in mitral and aortic regurgitation.J Heart Valve Dis. 1995;4(Suppl 2):SI60-8; discussion SI68-9.

\section{Correspondencia}

Dr. Alex Gallegos-Cazorla gallegoscazorla@hotmail.com

Fecha de recepción: 21 de agosto de 2014

Fecha de aceptación: 3 de junio de 2015

\section{Conflictos de interés}

El autor declara no tener conflictos de interés durante el planteamiento, ejecución de la investigación y la elaboración del artículo para su publicación. 